\title{
基于光波导分光光谱技术研究蛋白质与亚甲基蓝的竞争吸附行为
}

\author{
邓琳衩志美 * \\ (中国科学院电子学研究所, 传感技术国家重点实验室, 北京 100190)
}

\begin{abstract}
摘要：通过利用时间分辨光波导分光光谱技术原位测量从蛋白质-亚甲基蓝(MB)混合水溶液吸附到亲水玻璃 光波导表面的 MB 可见光吸收谱, 观测到在溶液 $\mathrm{pH}$ 值低于蛋白质等电点时 MB 与牛血清蛋白(BSA)以及 MB 与血红蛋白 $(\mathrm{Hb})$ 存在竞争吸附行为, 进一步测得这种竞争吸附行为对蛋白质浓度十分敏感, 可以用于简单测定 溶液中的蛋白质含量. 基于 Langmuir 等温吸附理论推导出了两种分子竞争吸附的动力学方程, 并利用该动力学 方程对实验测得的吸光度随时间变化曲线进行了最佳拟合, 揭示了玻璃表面吸附的 $\mathrm{MB}$ 分子个数在达到最大值 后随时间呈指数衰减, 同时得出拟合参数与蛋白质浓度呈准线性关系.
\end{abstract}

关键词：光波导分光光谱技术；竞争吸附；亚甲基蓝；蛋白质探测 中图分类号：0647

\section{Study of Competitive Adsorption Behavior of Protein and Methylene Blue by Optical Waveguide Spectroscopy}

\author{
DENG Lin QI Zhi-Mei* \\ (State Key Laboratory of Transducer Technology, Institute of Electronics, Chinese Academy of Sciences, \\ Beijing 100190, P. R. China)
}

\begin{abstract}
We used time-resolved optical waveguide spectroscopy (OWGS) to in-situ measure visible absorption spectrum for methylene blue (MB) adsorbed on a hydrophilic glass waveguide from an aqueous solution containing proteins such as bovine serum albumin (BSA) and hemoglobin $(\mathrm{Hb})$. The competitive adsorption of MB and the protein was detected at a solution $\mathrm{pH}$ lower than the isoelectric point of the protein. Since the competitive adsorption behavior of $\mathrm{MB}$ and the protein is very sensitive to the protein concentration, the protein content in the mixed solution was readily determined by time-resolved OWGS. In addition, a kinetic equation for the competitive adsorption of the two molecules was deduced based on the Langmuir adsorption isotherm. The best fit of the measured time course of the absorbance with the theoretical kinetic equation reveals that the number of MB molecules adsorbed on the surface exponentially decreases with time after reaching a maximum. A quasi-linear relationship between the fitting parameters and the protein concentration was also obtained.
\end{abstract}

Key Words: Optical waveguide spectroscopy; Competitive adsorption; Methylene blue; Protein detection

蛋白质作为生命的物质基础之一, 与各种形式 的生命活动紧密联系在一起, 它在催化生命体内的 各种反应, 调节新陈代谢以及控制遗传信息等方面 都起着至关重要的作用. 因此对于蛋白质的探测在
临床诊断、食品安全、生命现象研究等方面都有重要 的应用价值. 传统的探测蛋白质含量的方法主要有 Folin-酚试剂法(Lowry 法)、双缩艮法(Biuret 法)、凯 氏定氮法(Kjeldahl 法)、考马斯亮蓝法(Bradford 法)

Received: May 24, 2010; Revised: July 5, 2010; Published on Web: August 17, 2010.

*Corresponding author. Email: zhimei-qi@mail.ie.ac.cn; Tel: +86-10-58887533.

The project was supported by the National Key Basic Research Program of China (973) (2009CB320300), National Natural Science Foundation of China (60978042), and the BaiRenJiHua Program of Chinese Academy of Sciences.

国家重点基础研究发展计划(973)(2009CB320300), 国家自然科学基金(60978042) 和中科院“百人计划”择优支持项目资助

C Editorial office of Acta Physico-Chimica Sinica 
等 ${ }^{[1-6]}$, 操作时需要对待测样品进行复杂的化学前处 理过程.

随着集成光学技术的发展和波导制造技术的日 益成熟, 基于光波导传感技术的蛋白质探测方法越 来越受到人们的关注 ${ }^{[7-13]}$. 这类方法具有灵敏度高、 抗电磁干扰、轻便易集成等优点, 但由于蛋白质分子 通常在可见光波段是无色的, 直接探测比较困难, 通 常需要借助于抗原抗体配对或苂光标记等辅助处理 过程, 使得整个测试过程比较繁琐. 本文利用亚甲基 蓝(MB)作为吸附指示剂, 采用时间分辨光波导分光 光谱技术 ${ }^{[14-20]}$ 实时监测蛋白质与 $\mathrm{MB}$ 在亲水玻璃光 波导表面的竞争性吸附, 通过探测 MB 对导波光的 吸收来间接探测溶液中的蛋白质. 该方法操作简便, 样品需求量极少, 无需其它任何标记物, 只要选择合 适的缓冲溶液就可以检测各类蛋白质. 下文对牛血 清蛋白(BSA)和血红蛋白 $(\mathrm{Hb})$ 的探测证实了这种方 法的可行性, 而且其探测的灵敏度可以达到 $10^{-8}$ $\mathrm{mol} \cdot \mathrm{L}^{-1}$ 量级.

\section{1 实验部分}

\section{1 实验仪器及试剂}

LS-1-LL 型卤铇灯、光纤、HR4000 型 CCD 光谱 分析仪(美国 Ocean Optics 公司); $50 \mu \mathrm{m}$ 厚平面玻璃 光波导(日本 Matsunami Glass 公司); BQ50-1J 型蠕 动葲(中美合资保定兰格恒流洜有限公司); HARKESPCA 接触角测量仪 (北京哈科试验仪器厂); THB2000B 型 $\mathrm{pH}$ 计(上海宏胜集团); 玻璃棱镜、透镜、线 性偏振片(国产).

亚甲基蓝(MB)、磷酸、四硼酸钠(分析纯, 北京化 学试剂公司); 牛血清蛋白(BSA)、血红蛋白(Hb)(美 国 Sigma 公司).

\section{2 竞争吸附的探测方法}

每种蛋白质都有其特定的等电点(PI), 如果溶液 的 $\mathrm{pH}$ 值在蛋白质的等电点之上, 那么蛋白质分子 在溶液中带负电, 反之则带正电, 而亚甲基蓝(分子 式为 $\left[\mathrm{C}_{16} \mathrm{H}_{18} \mathrm{~N}_{3} \mathrm{~S}\right]^{+} \mathrm{Cl}^{-}$) 为阳离子染料, 在水溶液中电离

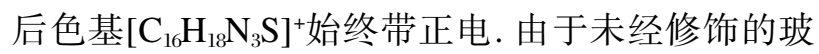
璃光波导表面显负电性, 如果调节溶液的 $\mathrm{pH}$ 值到 蛋白质的等电点之下, 那么蛋白质分子与 MB 分子 在光波导表面形成竞争吸附的关系. 通常蛋白质分 子体积较大, 吸附慢但吸附牢固, 而染料分子小, 吸 附快但吸附不牢固, 因此混合溶液的吸光度应该是 随时间迅速上升然后又逐渐下降至平衡的过程. 如
果选取吸收谱峰值处的某一波长(本文均采用 $\lambda=605$ $\mathrm{nm}$ )处的吸光度数据进行观察, 可以发现对于某种 特定的蛋白质, 不同的浓度对应不同的吸光度下降 速率; 而对于同一浓度的蛋白质, 在不同 $\mathrm{pH}$ 值的缓 冲溶液中, 对应的吸光度下降速率也不同, 这是本文 探测溶液中蛋白质含量的主要依据.

实验时首先用去离子水配制浓度为 $1 \mathrm{mmol} \cdot \mathrm{L}^{-1}$ 的标准 $\mathrm{MB}$ 溶液和浓度分别为 $1,2,5$ 和 $10 \mu \mathrm{mol} \cdot$ $\mathrm{L}^{-1}$ 的 BSA 溶液及 $\mathrm{Hb}$ 溶液备用. 对于 BSA, 配制了 $\mathrm{pH}$ 值分别为 2.03 和 4.02 的缓冲溶液, 溶液的 $\mathrm{pH}$ 值使用 $\mathrm{pH}$ 计测得, 可精确到小数点后两位. 本实验 中使用的去离子水的 $\mathrm{pH}$ 值为 5.89 , 磷酸经去离子 水稀释后用来提供 $\mathrm{pH}$ 值小于去离子水的各种缓冲 溶液. 对于 $\mathrm{Hb}$, 配制了 $\mathrm{pH}$ 值分别为 4.02 和 7.70 的 缓冲溶液, 其中 $\mathrm{pH}$ 值为 7.70 的缓冲溶液由四嗍酸 钠配制得到.

测试所用的溶液是向 $2.5 \mathrm{~mL}$ 缓冲溶液中滴人 $50 \mu \mathrm{L} 1 \mathrm{mmol} \cdot \mathrm{L}^{-1} \mathrm{MB}$ 备用溶液和 $100 \mu \mathrm{L}$ 蛋白质备 用溶液. 因此最终得到的待测溶液中, $M B$ 的浓度为 $18.868 \mu \mathrm{mol} \cdot \mathrm{L}^{-1}, \mathrm{BSA}$ 及 $\mathrm{Hb}$ 的浓度分别为 0.038 , $0.075,0.189$ 和 $0.377 \mu \mathrm{mol} \cdot \mathrm{L}^{-1}$. 为了准确观察蛋白 质浓度对吸光度的影响, 每次实验中 $\mathrm{MB}$ 的浓度保 持不变.

采用时间分辨光波导分光光谱法探测蛋白质和 $\mathrm{MB}$ 混合缓冲溶液在亲水玻璃表面的竞争吸附行为, 探测装置及操作方法在参考文献[20]中已有详细的 论述.

\section{2 结果与讨论}

\section{$2.1 \mathrm{BSA}$ 与 MB 的竞争吸附结果}

BSA 是血液的主要成分, 相对分子质量为 $66000 \mathrm{Da}$, 等电点为 4.7 . 为了对比实验结果, 首先以 去离子水 $(\mathrm{pH}=5.89)$ 为缓冲溶液进行实验, 由于去离 子水的 $\mathrm{pH}$ 值高于 $\mathrm{BSA}$ 的等电点, 因此观察不到竞 争吸附现象. 图 1 显示了 $\mathrm{pH}=5.89$ 的缓冲溶液中 $\mathrm{MB}$ 和 BSA 的共吸附光谱随时间的变化, 插图给出 了 $\lambda=605 \mathrm{~nm}$ 处的吸光度随时间的变化, 其中 BSA 的浓度为 $0.377 \mu \mathrm{mol} \cdot \mathrm{L}^{-1}, \mathrm{MB}$ 的浓度为 18.868 $\mu \mathrm{mol} \cdot \mathrm{L}^{-1}$.

下面分别采用 $\mathrm{pH}$ 值为 2.03 和 4.02 的缓冲溶 液进行实验, BSA 的浓度分别为 $0.038,0.075,0.189$ 和 $0.377 \mu \mathrm{mol} \cdot \mathrm{L}^{-1}$, 测试结果如图 2 和图 3 所示. 由 于滴人溶液的时刻比 $\mathrm{CCD}$ 开始记录数据的时刻有 


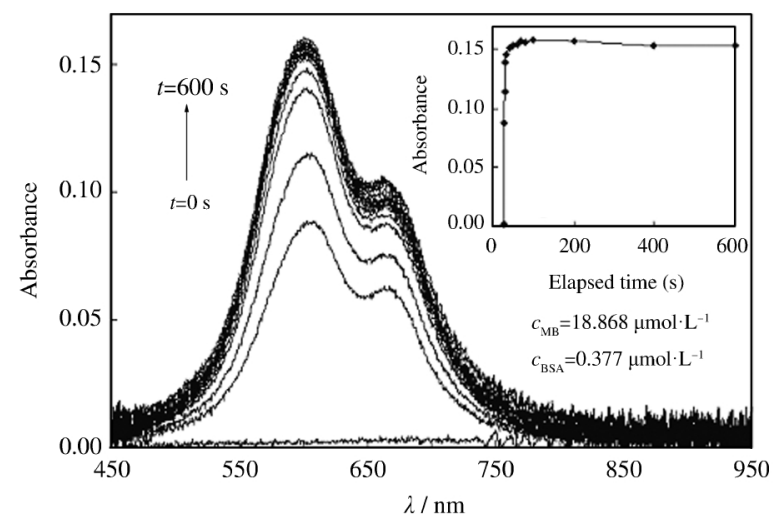

图 1 利用 pH 5.89 的 MB-BSA 混合水溶液得到的 MB 吸 附层的光波导吸收光谱

Fig.1 OWG absorption spectra of MB adsorbed from the pH 5.89 mixed aqueous solution of MB and BSA

Insert shows the absorbance at $\lambda=605 \mathrm{~nm}$ versus time.

所延迟, 所以吸光度曲线不是严格从 $t=0 \mathrm{~s}$ 开始上 升. 图 2 和图 3 中的曲线反映出 BSA 分子与亚甲基 蓝之间具有明显的竞争吸附现象, 最初亚甲基蓝迅 速吸附到光波导表面, 吸光度迅速上升至峰值, 之后 BSA 分子开始吸附并使得亚甲基蓝从光波导表面 脱附, 吸光度逐渐下降, 最终达到平衡. 对于某一特 定 $\mathrm{pH}$ 值的缓冲溶液, BSA 浓度越高, 吸光度下降越 快, 说明竞争吸附现象越明显.

为了讨论光波导芯片的可重复性使用, 每进行
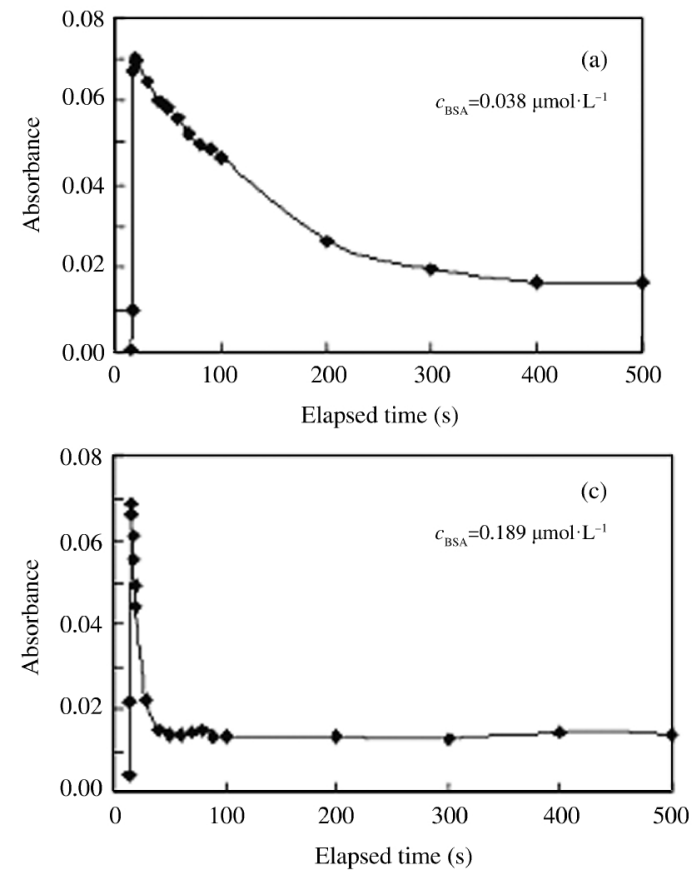

表 1 在各个实验阶段测得的玻璃光波导表面的水接触角 Table 1 Water contact angles of the glass waveguide surface measured at different time points during the experiment

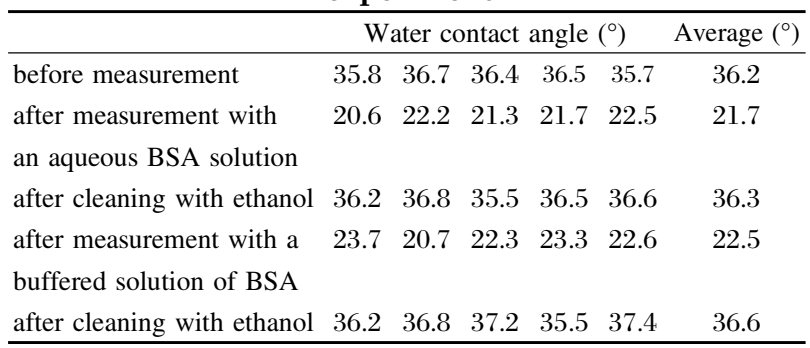

一步实验都会记录光波导表面五个不同位置的水接 触角变化, 每次实验完毕使用酒精仔细擦拭玻璃光 波导表面, 记录结果列于表 1 . 从表中数据可以看出, 酒精清洗可使光波导的水接触角恢复到实验前的水 平, 重复性良好.

\section{$2.2 \mathrm{Hb}$ 与 $\mathrm{MB}$ 的竞争吸附结果}

$\mathrm{Hb}$ 是高等生物体内负责运载氧的一种蛋白质, 它的等电点为 6.8 , 分子量为 $68000 \mathrm{Da}$. 对于 $\mathrm{Hb}$ 我 们同样测试了 $0.038,0.075,0.189$ 和 $0.377 \mu \mathrm{mol} \cdot \mathrm{L}^{-1}$ 四个浓度的蛋白质溶液. 由于 $\mathrm{Hb}$ 的等电点高于去 离子水的 $\mathrm{pH}$ 值, 因此实验用的缓冲溶液的 $\mathrm{pH}$ 值有 所调整, 分别采用了磷酸配制的 $\mathrm{pH}=4.02$ 的缓冲溶 液和四嗍酸钠配制的 $\mathrm{pH}=7.70$ 的缓冲溶液. 图 4 和 图 5 给出了这两种 $\mathrm{pH}$ 条件下的测试结果, 在 $\mathrm{pH}=$
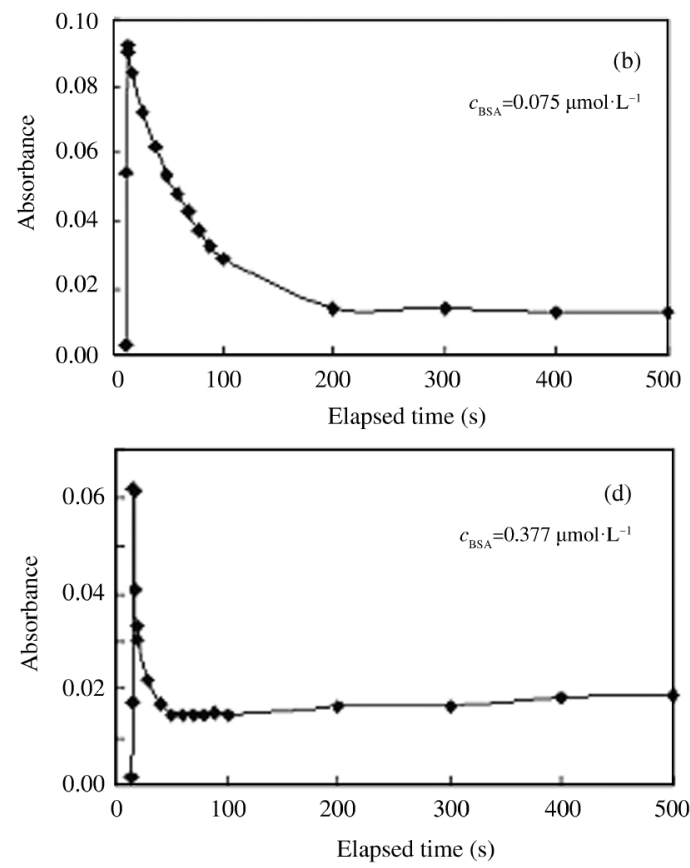

图 2 利用 pH 2.03 的 MB-BSA 混合水溶液得到的 $\mathrm{MB}$ 吸附层在 $\lambda=605 \mathrm{~nm}$ 处的光波导吸光度随时间的变化

Fig.2 Time courses of OWG absorbance at $\lambda=605 \mathrm{~nm}$ of $\mathrm{MB}$ adsorbed from the $\mathrm{pH} 2.03$ mixed aqueous solutions of MB and BSA 

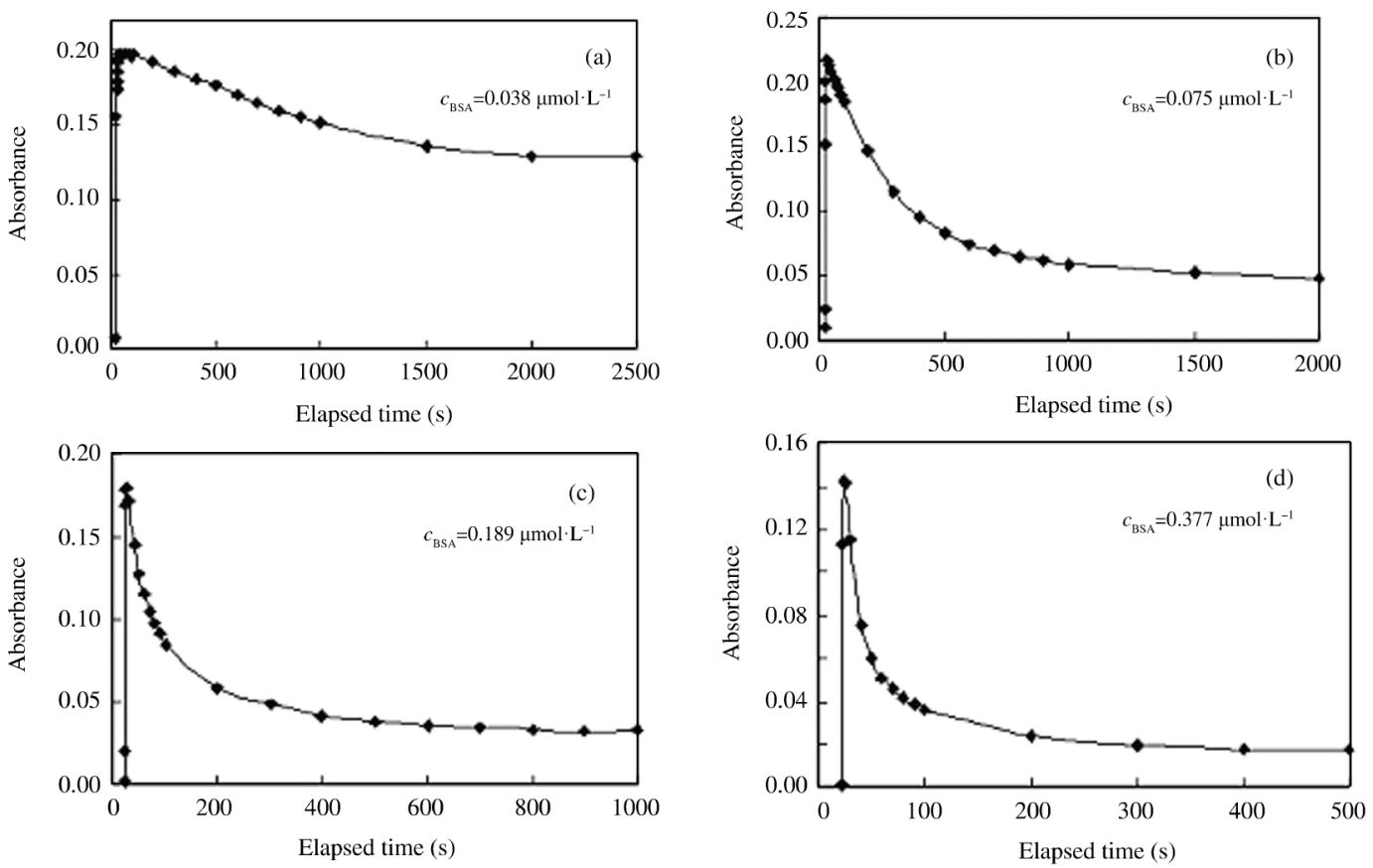

图 3 利用 $\mathrm{pH} 4.02$ 的 MB-BSA 混合水溶液得到的 $\mathrm{MB}$ 吸附层在 $\lambda=605 \mathrm{~nm}$ 处的光波导吸光度随时间的变化

Fig.3 Time courses of OWG absorbance at $\lambda=605 \mathrm{~nm}$ of $\mathrm{MB}$ adsorbed from the $\mathrm{pH} 4.02$ mixed aqueous solutions of MB and BSA

4.02 的缓冲溶液中可以观察到明显的竞争吸附现 象, 而在 $\mathrm{pH}=7.70$ 的缓冲溶液中则无法观察到竞争 吸附现象, 这跟所预期的结果相一致.

\section{3 竞争吸附的动力学模型}

光波导表面蛋白质亚单分子层和 $\mathrm{MB}$ 亚单分 子层的吸附都满足 Langmuir 吸附模型 ${ }^{[21-25]}$, 这里讨 论两种不同分子的竞争吸附动力学模型.

对于 $\mathrm{MB}$ 分子和蛋白质分子, 其吸附动力学方 程可以表示为式(1)和(2):

$$
\begin{aligned}
& \frac{\mathrm{d} N_{1}}{\mathrm{~d} t}=k_{\mathrm{a} 1} \frac{c_{1}}{55.5}\left(N_{\max }-N_{1}-N_{2}\right)-k_{\mathrm{d} 1} N_{1} \\
& \frac{\mathrm{d} N_{2}}{\mathrm{~d} t}=k_{\mathrm{a} 2} \frac{c_{2}}{55.5}\left(N_{\max }-N_{1}-N_{2}\right)-k_{\mathrm{d} 2} N_{2}
\end{aligned}
$$

其中, $N_{1}$ 和 $N_{2}$ 分别是某一时刻 $\mathrm{MB}$ 分子和蛋白质 分子的表面覆盖率, $N_{\max }$ 是最大表面覆盖率, $k_{\mathrm{a} 1} 、 k_{\mathrm{d} l}$ 和 $k_{\mathrm{a} 2} 、 k_{\mathrm{d} 2}$ 分别是 $\mathrm{MB}$ 和蛋白质的吸附、脱附速率常 数, $c_{1}$ 和 $c_{2}$ 分别为 $\mathrm{MB}$ 和蛋白质的体溶液浓度, 55.5 为水的浓度 $\left(\mathrm{mol} \cdot \mathrm{L}^{-1}\right)$.

当吸光度在时刻 $t_{\mathrm{m}}$ 达到最大值时, 图 2-图 4 中 的曲线达到拐点, 即 $\mathrm{d} N_{\mathrm{l}} / \mathrm{d} t=0$, 得到 $\mathrm{MB}$ 的覆盖率峰 值 $\left(N_{\text {lpeak }}\right)$ 为:

$$
N_{\text {lpeak }}=\frac{c_{1}\left(N_{\max }-N_{2}\right)}{c_{1}+55.5 \frac{k_{\mathrm{dl}}}{k_{\mathrm{al}}}}
$$

当 $N_{1}$ 达到峰值时, 可以认为 $\mathrm{d} N_{2} / \mathrm{d} t=-\mathrm{d} N_{1} / \mathrm{d} t$, 由此得 到:

$$
\begin{gathered}
N_{2}=-\frac{k_{\mathrm{al} 1} \frac{c_{1}}{55.5}+k_{\mathrm{a} 2} \frac{c_{2}}{55.5}+k_{\mathrm{d} 1}}{k_{\mathrm{a} 1} \frac{c_{1}}{55.5}+k_{\mathrm{a} 2} \frac{c_{2}}{55.5}+k_{\mathrm{d} 2}} N_{1}+ \\
\\
\frac{k_{\mathrm{a} 1} \frac{c_{1}}{55.5}+k_{\mathrm{a} 2} \frac{c_{2}}{55.5}}{k_{\mathrm{al}} \frac{c_{1}}{55.5}+k_{\mathrm{a} 2} \frac{c_{2}}{55.5}+k_{\mathrm{d} 2}} N_{\max }
\end{gathered}
$$

令 $\alpha=-\frac{k_{\mathrm{a} 1} c_{1}+k_{\mathrm{a} 2} c_{2}+55.5 k_{\mathrm{d} 1}}{k_{\mathrm{a} 1} c_{1}+k_{\mathrm{a} 2} c_{2}+55.5 k_{\mathrm{d} 2}}, \beta=\frac{k_{\mathrm{a} 1} c_{1}+k_{\mathrm{a} 2} c_{2}}{k_{\mathrm{a} 1} c_{1}+k_{\mathrm{a} 2} c_{2}+55.5 k_{\mathrm{d} 2}}$, 则 $N_{2}=\alpha N_{1}+\beta N_{\text {max }}$, 将 $N_{2}$ 代人式(1), 得到:

$$
\frac{\mathrm{d} N_{1}}{\mathrm{~d} t}=\frac{c_{1} k_{\mathrm{al}} N_{\max }}{55.5}(1-\beta)-\left[\frac{c_{\mathrm{l}} k_{\mathrm{al}}}{55.5}(1+\alpha)+k_{\mathrm{dl}}\right] N_{1}
$$

将式(5)移项后两端积分, 得到:

$$
\begin{aligned}
\int_{N_{\text {leak }}}^{N_{\mathrm{lt}}}\left\{\frac{c_{1} k_{\mathrm{al}} N_{\max }}{55.5}(1-\beta)-\left[\frac{c_{\mathrm{l}} k_{\mathrm{al}}}{55.5}(1+\alpha)+k_{\mathrm{dl}}\right] N_{\mathrm{l}}\right\}^{-1} \mathrm{~d} N_{\mathrm{l}}= \\
N_{\mathrm{l}}=\frac{c_{1} N_{\max }(1-\beta)}{c_{1}(1+\alpha)+55.5 \frac{k_{\mathrm{dl}}}{k_{\mathrm{al}}}}+\left[N_{\mathrm{lpeak}}-\frac{c_{1} N_{\max }(1-\beta)}{c_{1}(1+\alpha)+55.5 \frac{k_{\mathrm{dl}}}{k_{\mathrm{al}}}}\right] . \\
\quad \exp \left\{-\left[\frac{c_{1} k_{\mathrm{al}}}{55.5}(1+\alpha)+k_{\mathrm{dl}}\right]\left(t-t_{\mathrm{m}}\right)\right\}
\end{aligned}
$$



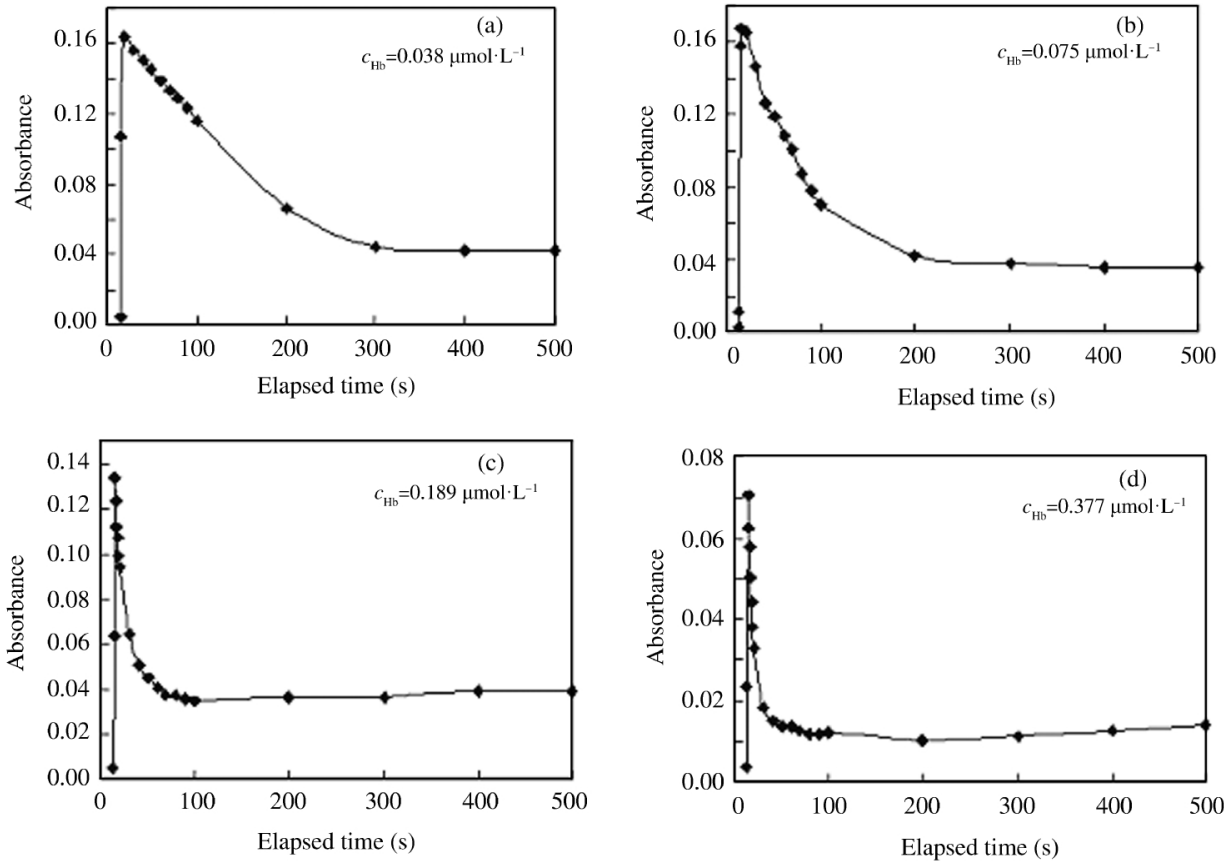

图 4 利用 $\mathrm{pH} 4.02$ 的 $\mathrm{MB}-\mathrm{Hb}$ 混合水溶液得到的 $\mathrm{MB}$ 吸附层在 $\lambda=605 \mathrm{~nm}$ 处的光波导吸光度随时间的变化

Fig.4 Time courses of OWG absorbance at $\lambda=605 \mathrm{~nm}$ of $\mathrm{MB}$ adsorbed from the $\mathrm{pH} 4.02$ mixed aqueous solutions of $\mathrm{MB}$ and $\mathrm{Hb}$

令 $\eta=\frac{c_{1} N_{\max }(1-\beta)}{c_{1}(1+\alpha)+55.5 \frac{k_{\mathrm{dl}}}{k_{\mathrm{al}}}}, \tau^{-1}=k_{\mathrm{al}} \frac{c_{1}}{55.5}(1+\alpha)+k_{\mathrm{dl}}$,

公式(7)可写为:

$$
N_{1 \tau}=\eta+\left(N_{\text {lpeak }}-\eta\right) \exp \left(-\frac{t-t_{\mathrm{m}}}{\tau}\right)
$$
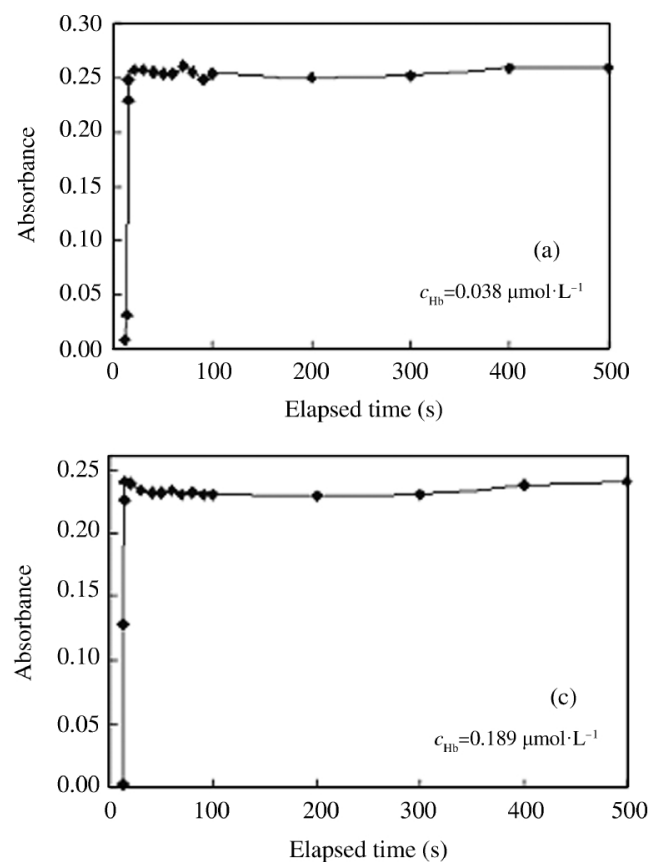

根据公式(8)可知, 在竞争吸附过程中 MB 的表面覆 盖率随时间的变化满足指数衰减关系. 公式(8)还指 出当时间趋于无穷大时 $N_{1}=\eta$, 也就是说在竞争吸附 达到平衡时 $\mathrm{MB}$ 的表面覆盖率为 $\eta$. 在上面的式子 中, $\alpha 、 \beta 、 \eta, \tau$ 都是只与 $k_{\mathrm{a} 1} 、 k_{\mathrm{d} 1} 、 k_{\mathrm{a} 2} 、 k_{\mathrm{d} 2} 、 c_{1} 、 c_{2} 、 N_{\max }$ 有关
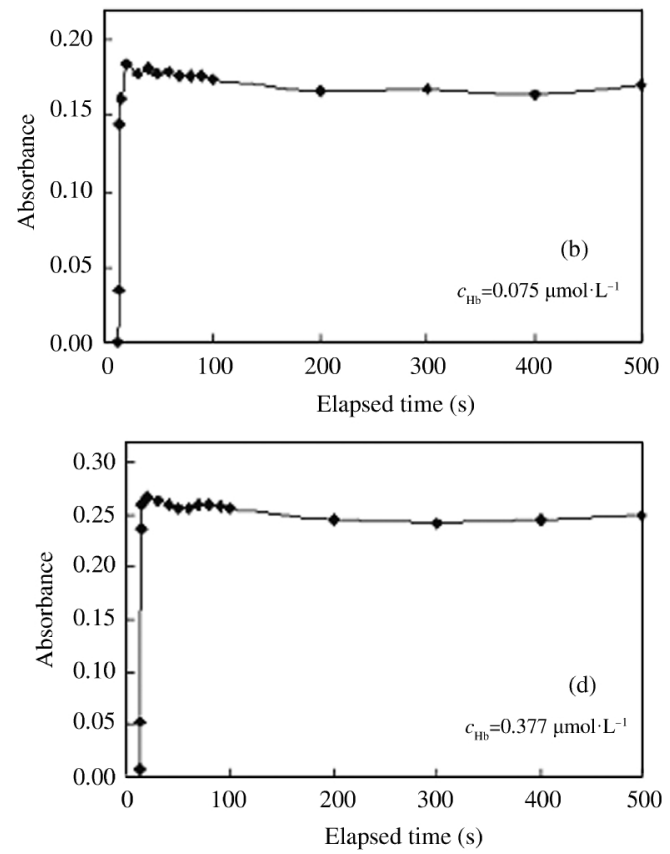

图 5 利用 $\mathrm{pH} 7.70$ 的 $\mathrm{MB}-\mathrm{Hb}$ 混合水溶液得到的 $\mathrm{MB}$ 吸附层在 $\lambda=605 \mathrm{~nm}$ 处的光波导吸光度随时间的变化

Fig. 5 Time courses of $\mathrm{OWG}$ absorbance at $\lambda=605 \mathrm{~nm}$ of $\mathrm{MB}$ adsorbed from the $\mathrm{pH} 7.70$ mixed aqueous solutions of $\mathrm{MB}$ and $\mathrm{Hb}$ 


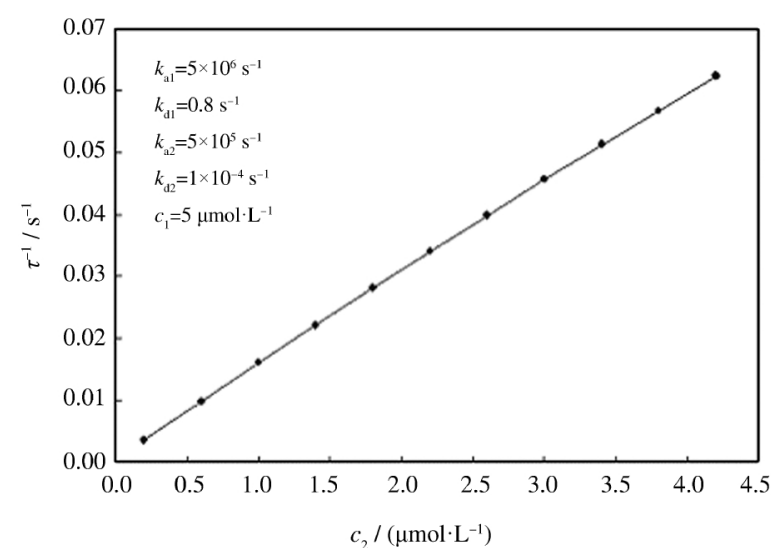

图 6 模拟计算得出的拟合参数 $\tau^{-1}$ 与蛋白质浓度的线性关系

Fig.6 Linear relationship between the fitting parameter $\tau^{-1}$ and protein concentration obtained with simulation

$k$ : rate constant, subscripts 1 and 2 denote the MB and protein, respectively, and subscripts a and $\mathrm{d}$ refer to adsorption and desorption, respectively.

的常数.

根据光波导消逝场理论分析可知在时刻 $t$ 测得 的给定波长的吸光度 $\left(A_{t}\right)$ 与该时刻的 $\mathrm{MB}$ 表面覆盖 率成正比, 即 $A_{t}=\gamma N_{\mathrm{l} t}, A_{\text {max }}=\gamma N_{\mathrm{lpeak}}, A_{\text {eq }}=\gamma \eta$, 其中 $\gamma$ 为 比例常数, $A_{\text {max }}$ 为峰值吸光度, $A_{\mathrm{eq}}$ 是竞争吸附达到 平衡时的吸光度. 因此由公式(8)可以得出公式(9):

$$
A_{t}=A_{\text {eq }}+\left(A_{\text {max }}-A_{\text {eq }}\right) \exp \left(-\frac{t-t_{\mathrm{m}}}{\tau}\right)
$$

在公式(8)和(9)中参数 $\tau$ 的单位为 $\mathrm{s}$, 其含义为 吸光度从其峰值下降了峰值与稳定值之差的 1/e 所 用的时间, 或者说 MB 覆盖率从其峰值下降了峰值 与稳定值之差的 $1 / \mathrm{e}$ 所用的时间. 给定 $k_{\mathrm{a} 1 、} k_{\mathrm{d} 1} 、 k_{\mathrm{a} 2} 、 k_{\mathrm{d} 2}$ 、

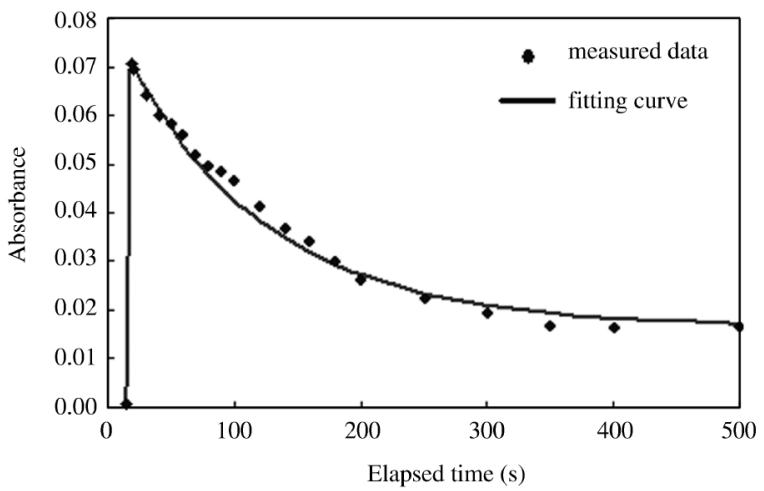

图 7 被吸附的 $M B$ 分子在 $\lambda=605 \mathrm{~nm}$ 处的吸光度随时间 的变化及其最佳拟合曲线

Fig.7 Best fitting curve of the time course of absorbance at $\lambda=605 \mathrm{~nm}$ for adsorbed MB molecules $c_{\mathrm{BSA}}=0.038 \mu \mathrm{mol} \cdot \mathrm{L}^{-1}, c_{\mathrm{MB}}=18.868 \mu \mathrm{mol} \cdot \mathrm{L}^{-1}, \mathrm{pH}=2.03$ $c_{1}$ 和 $N_{\text {max }}$, 利用公式(8)对 $\tau$ 和 $c_{2}$ 的依赖关系进行模 拟计算, 得到如图 6 所示的结果. 从图中可看出 $\tau$ 的 倒数 $\left(\tau^{-1}\right)$ 随着 $c_{2}$ 的增大而线性增加. 由此可见增大 蛋白质浓度能够使吸附竞争加剧, 使竞争吸附达到 平衡所用的时间缩短. 在利用公式(9)对图 2-图 4 中 的实验曲线进行拟合时把 $\tau^{-1}$ 直接作为拟合参数. 为了验证拟合效果, 选取图 2(a)进行拟合, 得到图 7 所示的结果, 实验曲线与拟合曲线吻合良好, 说明上 述理论推导得出的拟合公式具有充分的合理性.

根据拟合的结果, 我们可以给出拟合参数 $\tau^{-1}$ 与 $\mathrm{BSA}$ 浓度及 $\mathrm{Hb}$ 浓度的关系曲线, 图 8(a)和(b)分别 给出了 $\mathrm{pH}=2.03$ 和 $\mathrm{pH}=4.02$ 时拟合参数 $\tau^{-1}$ 与 $\mathrm{BSA}$ 浓度的关系图. 图 9 给出了 $\mathrm{pH}=4.02$ 时拟合参数 $\tau^{-1}$ 与 $\mathrm{Hb}$ 浓度的关系图. 可以发现对于不同的 $\mathrm{pH}$ 条件 以及不同的蛋白质, 拟合参数 $\tau^{-1}$ 随蛋白质浓度的升 高而增大, 二者呈准线性关系, 这与图 6 给出的模拟 计算结果一致.
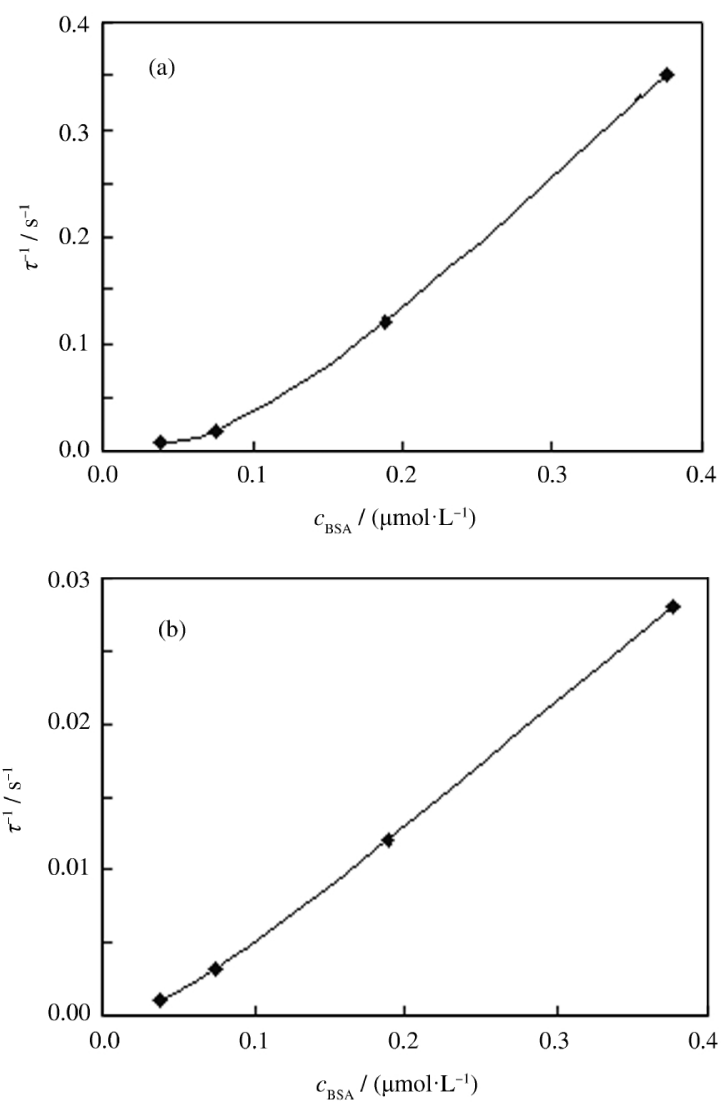

图 8 (a) $\mathrm{pH}=2.03$ 和 $(\mathrm{b}) \mathrm{pH}=4.02$ 时拟合参数 $\tau^{-1}$ 与 $\mathrm{BSA}$ 浓度的关系

Fig.8 Relationships between the fitting parameter $\tau^{-1}$ and BSA concentration obtained at (a) $\mathrm{pH}=2.03$ and (b) $\mathrm{pH}=4.02$ 


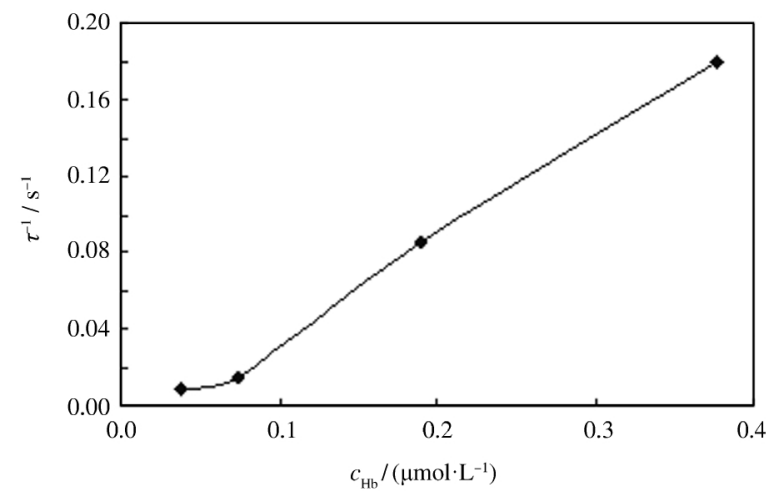

图 $9 \mathrm{pH}=4.02$ 时拟合参数 $\tau^{-1}$ 与 $\mathrm{Hb}$ 浓度的关系

Fig.9 Relationship between the fitting parameter $\tau^{-1}$ and $\mathrm{Hb}$ concentration obtained at $\mathrm{pH}=4.02$

\section{3 结 论}

本文研究了吸附指示剂 $\mathrm{MB}$ 与两种蛋白质 $\mathrm{BSA}$ 和 $\mathrm{Hb}$ 在亲水玻璃光波导表面的竞争吸附行 为, 给出了一种通过探测 $\mathrm{MB}$ 对导波光的吸收来间 接探测溶液中蛋白质含量的方法. 该方法基于光波 导分光光谱技术, 与其它基于光波导的探测技术相 比, 有效地避免了复杂的样品前处理过程, 如: 蛋白 质抗原抗体配对以及苂光标记等, 使得整个测试过 程简便易操作. 文中根据 Langmuir 吸附模型推导出 了两种分子竞争吸附的动力学方程, 根据推导结果 对实验测得的吸光度曲线进行拟合, 发现实验数据 与理论推导符合得很好. 由于竞争吸附的存在, MB 与蛋白质混合溶液的吸光度在达到峰值以后随时间 呈指数衰减, 对于某一特定的蛋白质, 当缓冲溶液的 $\mathrm{pH}$ 值一定时, 蛋白质浓度越高, 吸光度下降越快, 即 衰减系数越大. 考察不同情况下拟合参数与蛋白质 浓度的关系, 得到的曲线具有良好的规律性, 为溶液 中蛋白质含量的探测提供了依据.

\section{References}

1 Marco, A.; Rubio, R.; Compano, R.; Casals, I. Talanta, 2002, 57: 1019

2 Coakley, W. T.; James, C. J. Anal. Biochem., 1978, 85: 90

3 Shen, W.; Chen, J. Z.; Dai, C. J. Food Res. Dev., 2009, 30: 96 [沈 文, 陈均志, 代春吉. 食品研究与开发, 2009, 30: 96]
4 Li, J.; Zhang, Y. T.; Zeng, W.; Luo, X.; Liao, C. C. Chin. J. Biol., 2000, 13: 118 [李 娟, 张耀庭, 曾 伟, 罗 璇, 廖长春. 中国 生物制品学杂志, 2000, 13: 118]

5 Minagawa, M.; Winter, D. A.; Kaplan, I. R. Anal. Chem., 1984, 56: 1859

6 Penninckx, W.; Verbeke, J. S.; Vankeerberghen, P.; Massart, D. L. Anal. Chem., 1996, 68: 481

7 Yu, X. L.; Wei, X.; Wang, D. X.; Ding, X.; Liao, W.; Zhao, X. S. Acta Phys. -Chim. Sin., 2005, 21: 888 [余兴龙, 魏 星, 王鼎新, 定 翔, 廖 伟, 赵新生. 物理化学学报, 2005, 21: 888]

8 Schmitt, K.; Schirmer, B.; Hoffmann, C.; Brandenburg, A.; Meyrueis, P. Biosens. Bioelectro., 2007, 22: 2591

9 Wu, Z. W.; Liu, Q. J.; Wu, L. W.; Xie, X.; Zhuang, Y.; Wen, T.; Bai, Y. F.; Lu, Z. H. Acta Optica Sinica, 2007, 27: 521 [吴中伟, 刘全俊, 吴凌伟, 谢 骁, 庄 萤, 温 恬, 白云飞, 陆祖宏. 光学 学报, 2007, 27: 521]

10 Kim, N.; Kim, D. K.; Kim, W. Y. Food Chem., 2008, 108: 768

11 Enami, Y.; Fukuda, T.; Suye, S. Appl. Phys. Lett., 2007, 91: 203507-1

12 Kim, N.; Kim, D. Y.; Cho, Y. J.; Moon, D. K.; Kim, W. Y. Biosens. Bioelectro., 2008, 24: 391

13 Choquette, S. J.; Browm, L. L.; Durst, R. A. Anal. Chem., 1992, 64: 55

14 Bradshaw, J. T.; Mendes, S. B.; Saavedra, S. S. Anal. Chem., 2005 29A

15 Qi, Z. M.; Matsuda, N.; Takatsu, A.; Kato, K. J. Phys. Chem. B, 2003, 107: 6873

16 Cameron, P. J.; Jenkins, A. T. A.; Knoll, W.; Marken, F.; Milsom, E. V.; Williams, T. L. J. Mater. Chem., 2008, 18: 4304

17 Qi, Z. M.; Xia, S. H.; Matsuda, N. Anal. Biochem., 2008, 374: 196

18 Mendes, S. B.; Saavedra, S. S. Opt. Express, 1999, 4: 449

19 Fujita, K.; Taniguchi, K.; Ohno, H. Talanta, 2005, 65: 1066

20 Deng, L.; Lu, D. F.; Qi, Z. M. Acta Phys. -Chim. Sin., 2009, 25 2481 [邓 琳, 逯丹凤, 祁志美. 物理化学学报, 2009, 25: 2481]

21 Azizian, S.; Haerifar, M.; Parsa, J. B. Chemosphere, 2007, 68: 2040

22 Gomri, S.; Seguin, J. L.; Guerin, J.; Aguir, K. Sens. Actuators, 2006, 114: 451

23 Koutsopoulos, S.; Patzsch, K.; Bosker, W. T. E.; Norde, W. Langmuir, 2007, 23: 2000

24 Kusakari, A.; Izumi, M.; Ohnuki, H. Colloids Surf. A, 2008, 321 47

25 Can, O.; Holland, N. B. J. Colloid Interface Sci., 2009, 329: 24 